\title{
Guest Editorial: Intelligent solutions for green computing in internet of medical things
}

\author{
Alireza Souri ${ }^{1} \cdot$ Vincenzo Piuri ${ }^{2} \cdot \mathrm{Mu}-\mathrm{Yen}_{\mathrm{Chen}}{ }^{3}$ \\ Published online: 26 April 2021 \\ (c) The Author(s), under exclusive licence to Springer-Verlag GmbH Austria, part of Springer Nature 2021
}

\section{Introduction}

Internet of Medical Things (IoMT) is one of the important subjects of Internet of Things (IoT) that navigates data processing on medical devices, healthcare information, and medical sensors by obtaining, analysing, and sharing data over the internet. The IoMT environment selects just privacy-aware, secure and reliable IoT services before service selection and recommendation are performed. Therefore, safety-critical issues are needed because not all smart devices will be available, reliable and some IoMT devices may behave maliciously to disrupt the cloud service (e.g., an adversary) or just for their gain to provide requested services based on Service Level Agreement (SLA).

Because the medical devices monitor and process sensitive and high-risk personal health data, they should be much secured to protect the user's privacy. Due to heterogeneity and impotence of sensitive and critical medical information in healthcare applications, collecting, processing and transmitting them are essential to save and secure the IoMT environment from potential attacks and suspicious conditions.

By increasing applying intelligent medical and healthcare systems, secured green computing is incoming a more developed stage in the IoMT environment. Moreover, the sensitive and critical medical information sharing and humanized

Alireza Souri

a.souri@ srbiau.ac.ir

Vincenzo Piuri

vincenzo.piuri@unimi.it

Mu-Yen Chen

mychen119@gs.ncku.edu.tw

1 Department of Computer Engineering, Science and Research Branch, Islamic Azad Unviersity, Tehran, Iran

2 Department of Computer Science, University of Milan, Milan, Italy

3 Department of Engineering Science, National Cheng Kung University, Tainan, Taiwan intelligent recommendations provide recent green computing play sensitive and important role in the IoMT.

To this point, safety-critical solutions for green computing in IoMT systems have emerging efforts and high potential to evaluate the reliable points and safety conditions. The goal of this special issue is to highlight the latest research focusing on intelligent solutions for green computing in IoMT to address the challenges and critical points. This special issue finalized and collected various scientific contributions that seven research studies have been accepted with a peer-review process in Network Modelling Analysis in Health Informatics and Bioinformatics Computing journal.

This special issue finalized peer-reviewed original articles that present new efforts to solve new challenges of healthcare and medical systems in IoT environments. These contributions represent several different aspects of intelligent methods based on data mining, machine learning, swarm intelligent algorithms and linear programming for biological analysis, recognition of psychological emotion, and analysing electronic medical records in the IoT environments.

\section{Conclusion}

We would like to thank the Editor-in-Chief Prof. Uffe Kock Wiil and Prof. Reda Alhajj, editorial members and reviewers to support this special issue and judgement of high-quality papers that provide a state-of-the-art overview on the existing challenges and opportunities of the IoMT. Also, we would like to thank all authors for submitting their work to this special issue.

Publisher's Note Springer Nature remains neutral with regard to jurisdictional claims in published maps and institutional affiliations. 